\title{
QUASI-REGULARITY VERIFICATION FOR 2D POLYGONAL OBJECTS BASED ON MEDIAL AXIS ANALYSIS
}

\author{
Phuc Ngo $^{\star}$, Nicolas Passat ${ }^{\dagger}$ and Yukiko Kenmochi ${ }^{\ddagger}$ \\ * Université de Lorraine, LORIA, UMR 7503 Villers-lès-Nancy, France \\ $\dagger$ Université de Reims Champagne Ardenne, CReSTIC EA 3804, 51097 Reims, France \\ ${ }_{\ddagger}^{\ddagger}$ LIGM, Univ Gustave Eiffel, CNRS, ESIEE Paris, Marne-la-Vallée, France
}

\begin{abstract}
Quasi-regularity has been proved to be a sufficient condition for simple-connectedness preservation during the digitization process of $2 \mathrm{D}$ continuous objects. The original definition of quasi-regularity relies on set-based morphological operations of erosion and dilation. With this definition, quasi-regularity is algorithmically difficult to assess. In this paper, we propose a tractable framework for quasiregularity verification, especially designed for polygons. Our approach mainly relies on the computation and analysis of the medial axis of these objects, and determines their potential quasi-regularity, and thus their ability to undergo a digitization without alteration of their topological properties. The framework is applied in the context of topology-preserving rigid motions of digital objects.
\end{abstract}

Index Terms - Quasi-regularity, polygonal objects, medial axis, topology preservation, rigid motion.

\section{INTRODUCTION}

A digital object $X \subset \mathbb{Z}^{2}$ is often the result of a digitization process, namely sampling or quantification, applied on a continuous object $X \subset \mathbb{R}^{2}$. Because of this process, the resulting object may have different properties than those of the original continuous one. This article addresses topological properties of objects in the case of the Gauss digitization $\mathcal{D}[1]$. In this context, some studies were proposed for providing topological guarantees by $\mathcal{D}$. Pavlidis, in the 1980 s, can be considered as the pioneer of the topology-preserving conditions of digitized objects. More precisely, he introduced in [2] the notion of $r$-regularity, and established the topological links between a continuous shape and its digitized counterpart. However, he was interested in the "smooth" objects, i.e. with boundaries having differentiable properties. Later, Stelldinger and Terzic presented in [3] a generalization of $r$-regularity, named $r$-halfregularity, which includes objects with non-differentiable boundaries. It was shown that the $r$-halfregularity allows a topologically correct digitization using an additional repairing step. Recently, a morphology-based notion, called quasi-r-regularity, was proposed in [4] and further formalized in 2D in [5]. A preliminary extension in 3D was presented in [6]. This notion provides sufficient conditions for simpleconnectedness preservation by $\mathcal{D}$. It was presented together with a rigid motion model that allows to preserve topology and some geometrical properties of digitized objects.

However, no algorithmic framework was given for quasiregularity verification. Based on the relations between the quasiregularity and the notion of medial axis, we propose hereafter such framework, dedicated to polygonal objects, frequently used in image analysis or computer graphics. An application for topologypreserving rigid motion of digital objects is also proposed.
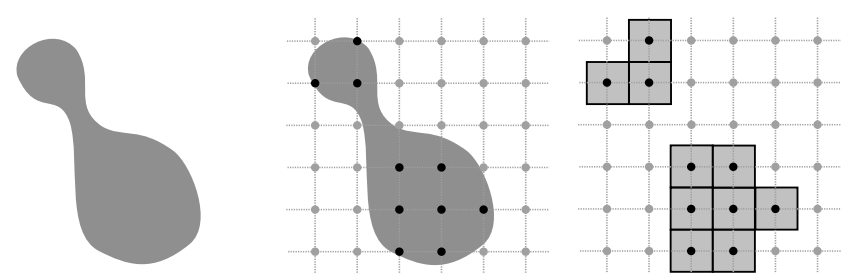

Fig. 1: From left to right: an object $X \subset \mathbb{R}^{2}$; its Gauss digitization, $\mathrm{X}=X \cap \mathbb{Z}^{2}$; and the digital object $\mathrm{X}$ represented by pixels. $X$ and $\mathrm{X}$ are not topologically equivalent ( $X$ is connected but $\mathrm{X}$ is not).

\section{PRELIMINARIES}

\subsection{Gauss digitization}

Let $X \subset \mathbb{R}^{n}, n \geq 2$. Digitization can be defined as a process of transforming $X$ into a discrete set. In this work, we consider the Gauss digitization [1], which is the intersection of $X$ with $\mathbb{Z}^{n}$ :

$$
\mathrm{X}=X \cap \mathbb{Z}^{n}
$$

The obtained object $X \subseteq \mathbb{Z}^{n}$ is called a digital object. It is a subset of $\mathbb{Z}^{n}$ (finite if $X$ is bounded). In 2D and from an imaging point of view, $X \subset \mathbb{Z}^{2}$ can be seen as a set of pixels, i.e. unit squares corresponding to the Voronoi cells of $\mathbb{Z}^{2}$ in $\mathbb{R}^{2}$. The structure of $X$ can be defined in various topological frameworks which are mainly equivalent [7] to that of digital topology [8]. Due to the approximations induced by the digitization process from $\mathbb{R}^{2}$ to $\mathbb{Z}^{2}$, the (digital) topology of $X$ is often not equivalent to the (continuous) topology of $X$. As illustrated in Fig. 1. when a 2D connected object is digitized, its connectedness is not always preserved.

\subsection{Quasi-regularity}

We now recall the notion of quasi-r-regularity [5] for objects whose boundaries are not necessarily differentiable.

Definition 1 (Quasi- $r$-regularity [5]) Let $r>0$. Let $X \subset \mathbb{R}^{2}$ be a bounded, simply connected object (i.e. connected without hole). $X$ is quasi-r-regular if it satisfies the following four properties:

(i) $X \ominus B_{r}$ is non-empty and connected

(ii) $\bar{X} \ominus B_{r}$ is connected

(iii) $X \subseteq X \ominus B_{r} \oplus B_{r \sqrt{2}}$

(iv) $\bar{X} \subseteq \bar{X} \ominus B_{r} \oplus B_{r \sqrt{2}}$

where $\bar{X}=\mathbb{R}^{2} \backslash X ; \oplus, \ominus$ are the dilation and erosion operators [9]; and $B_{t} \subset \mathbb{R}^{2}$ is the Euclidean ball of centre $0_{\mathbb{R}^{2}}$ and radius $t$. 
In practice, the value $r=1$ is used for considering quasi- $r$-regularity (then called quasi-regularity, for short). Quasi-regularity was proved to be a sufficient condition for preserving the simple connectedness of continuous object by the Gauss digitization.

Proposition 2 (|[5]) Let $X \subset \mathbb{R}^{2}$ be a bounded, simply connected object. If $X$ is quasi-regular, then $\mathrm{X}=X \cap \mathbb{Z}^{2}$ and $\overline{\mathrm{X}}=\bar{X} \cap \mathbb{Z}^{2}$ are both 4-connected.

The used notion of 4-connectedness usually considered in digital topology [8] is the equivalence relation derived from the 4 -adjacency in $\mathbb{Z}^{2}\left(\mathrm{p}, \mathrm{q} \in \mathbb{Z}^{2}\right.$ are 4 -adjacent if $\left.\|\mathrm{p}-\mathrm{q}\|_{1}=1\right)$.

Note that from Prop. 2 it derives that $X$ and $\bar{X}$ are not only simply connected, but also well-composed [10].

Quasi-regularity is then a sufficient condition for guaranteeing the topological preservation of a simply connected object when it undergoes a digitization process from $\mathbb{R}^{2}$ to $\mathbb{Z}^{2}$.

\subsection{Medial axis}

Let $X \subset \mathbb{R}^{2}$ be a closed, bounded set such that the boundary $\partial X$ of $X$ is a 1-manifold. We note $B(y, r)$ the Euclidean ball of center $y \in \mathbb{R}^{2}$ and radius $r \in \mathbb{R}_{+}$. Let $x \in X$. We set $r(x) \in \mathbb{R}_{+}$as the radius of the maximal Euclidean ball of center $x$, included in $X$ :

$$
r(x)=\max \{r \mid B(x, r) \subseteq X\}
$$

Blum defined the medial axis $\mathcal{M}(X)$ of $X$ as the set of points in $X$ having more than one closest point on its boundary $\partial X$ [11]:

$$
\mathcal{M}(X)=\{x \in X|| B(x, r(x)) \cap \partial X \mid>1\}
$$

Alternatively, the medial axis of $X$ can be defined as the locus of the centers of the maximal balls included in $X$ :

$$
\mathcal{M}(X)=\{x \in X \mid \nexists y \in X, B(x, r(x)) \subset B(y, r(y))\}
$$

The slight difference between these two definitions lies in the case of non-differentiable points of $\partial X$. For such points, the maximal Euclidean ball has a null radius. As a consequence, these points belong to the medial axis w.r.t Eq. (4) but not w.r.t Eq. (3). However, both medial axes are equal up to closure.

From the very definition of medial axis, we have $\mathcal{M}(X) \subseteq X$ and:

$$
X=\bigcup_{x \in \mathcal{M}(X)} B(x, r(x))
$$

In other words, $X$ can be reconstructed from $\mathcal{M}(X)$. We now define from $\mathcal{M}(X)$ the $\lambda$-level medial axis, noted $\mathcal{M}_{\lambda}(X)$, as:

$$
\mathcal{M}_{\lambda}(X)=\{x \in \mathcal{M}(X) \mid r(x) \geq \lambda\}
$$

In particular, we have $\lambda_{1} \leq \lambda_{2} \Rightarrow \mathcal{M}_{\lambda_{2}}(X) \subseteq \mathcal{M}_{\lambda_{1}}(X)$, and $\mathcal{M}_{0}(X)=\mathcal{M}(X)$. We can also define:

$$
\mathcal{M}_{\lambda_{1}}^{\lambda_{2}}(X)=\left\{x \in \mathcal{M}(X) \mid \lambda_{1} \leq r(x) \leq \lambda_{2}\right\}
$$

We state two important results that will be used in the sequel.

Proposition 3 ([12]) $X$ and $\mathcal{M}(X)$ have the same homotopy type.

In [12], the property is established for $X$ open. Here, it remains valid for $X$ closed, since we assume that $\partial X$ is a manifold. From now on, we denote $X \frown Y$ if $X$ and $Y$ have the same homotopy type.

Proposition 4 ([9]) Let $B_{\lambda}$ be the Euclidean ball of centre $0_{\mathbb{R}^{2}}$ and radius $\lambda \geq 0$. We have:

$$
\begin{aligned}
X \ominus B_{\lambda} & =\bigcup_{x \in \mathcal{M}_{\lambda}(X)} B(x, r(x)-\lambda) \\
X \oplus B_{\lambda} & =\bigcup_{x \in \mathcal{M}(X)} B(x, r(x)+\lambda) \\
\mathcal{M}\left(X \ominus B_{\lambda}\right) & =\mathcal{M}_{\lambda}(X)
\end{aligned}
$$

\section{QUASI-REGULARITY WITH MEDIAL AXIS}

The above notions are valid for general objects of $\mathbb{R}^{2}$. From now on, we focus on polygonal objects. Our purpose is to assess the quasiregularity of such objects, in order to know if they may preserve their topological properties when undergoing a Gauss digitization. Such verification is indeed difficult based on Def. 1. in particular since conditions $(i i i)$ and $(i v)$ require to compute the erosions and dilations of sets and to compare them with respect to inclusion. To tackle this issue, we propose to rely on the medial axis as a way to model these polygonal objects. Using this paradigm, we present hereafter a way of assessing the quasi-regularity of a polygonal object, thus leading to a tractable algorithm (Sec. 4).

Property 5 Let $X \subset \mathbb{R}^{2}$ be a bounded, simply connected polygon. If $\mathcal{M}(X) \frown \mathcal{M}_{1}(X)$ and $\mathcal{M}(\bar{X}) \frown \mathcal{M}_{1}(\bar{X})$ then conditions $(i)$ and (ii) of Def. 1 hold.

Proof Let us suppose that $\mathcal{M}(X) \frown \mathcal{M}_{1}(X)$. We have $X \frown$ $\mathcal{M}(X)$ (Prop. 3), $\mathcal{M}(X) \frown \mathcal{M}_{1}(X)$ by hypothesis, $\mathcal{M}_{1}(X)=$ $\mathcal{M}\left(X \ominus B_{1}\right)$ (Eq. [10), and $\mathcal{M}\left(X \ominus B_{1}\right) \frown X \ominus B_{1}$ (Prop. 3). Since $X$ is non-empty and simply connected by hypothesis, it follows that $X \ominus B_{1}$ is non-empty and connected; then $(i)$ holds. If we assume $\mathcal{M}(\bar{X}) \frown \mathcal{M}_{1}(\bar{X})$, the same reasoning holds for $\bar{X}$ chosen sufficiently larg $\AA^{1}$ and connected.

In the sequel, $Y$ can be either $X$ or $\bar{X}$. Let us consider the object $Y \circ B_{1}=Y \ominus B_{1} \oplus B_{1}$, namely the opening of $Y$ by $B_{1}$. From Prop. 4 it is plain that:

$$
Y \circ B_{1}=\bigcup_{x \in \mathcal{M}_{1}(Y)} B(x, r(x)) \subseteq Y
$$

Let us define the top-hat of $Y$ as $T_{B_{1}}(Y)=Y \backslash\left(Y \circ B_{1}\right)$. From Eqs. (5) and (11), we have:

$$
T_{B_{1}}(Y) \subseteq \bigcup_{x \in \mathcal{M}_{0}^{1}(Y)} B(x, r(x))
$$

Let $M \subseteq \mathcal{M}_{0}^{1}(Y)$ be a connected component of $\mathcal{M}_{0}^{1}(Y)$. From Prop. 5, there exists a unique point $y \in M$ such that $r(y)=1$. Since $Y$ has a polygonal shape, the set $M$ contains $k$ terminal points $(k \geq 1)$, namely points $z_{i}(1 \leq i \leq k)$ such that $r\left(z_{i}\right)=0$. These points are convex vertices of the polygon $Y$.

We set $\mathcal{C}(M) \subset \mathbb{R}^{2}$ as the convex hull of $B(y, 1) \cup\left\{z_{i}\right\}_{i=1}^{k}$. Then, we have:

$$
\bigcup_{x \in M} B(x, r(x)) \subseteq \mathcal{C}(M)
$$

In particular, if we assume that $(\mathcal{P}) \forall 1 \leq i \leq k,\left\|y-z_{i}\right\|_{2} \leq \sqrt{2}$, it follows that $\mathcal{C}(M) \subseteq B(y, \sqrt{2})$. Since $y \in Y \ominus B_{1}$, we have:

$$
(\mathcal{P}) \Rightarrow \bigcup_{x \in M} B(x, r(x)) \subseteq Y \ominus B_{1} \oplus B_{\sqrt{2}}
$$

From Eqs. 12) and (14, we then obtain the following result.

Proposition 6 Let $X \subset \mathbb{R}^{2}$ be a bounded, simply connected polygon. Let us suppose that $\mathcal{M}(X) \frown \mathcal{M}_{1}(X), \mathcal{M}(\bar{X}) \frown \mathcal{M}_{1}(\bar{X})$ and that for each connected component of $\mathcal{M}_{0}^{1}(X)$ and $\mathcal{M}_{0}^{1}(\bar{X})$, property $(\mathcal{P})$ holds. Then, $X$ is quasi-regular.

\footnotetext{
${ }^{1}$ In order to handle the medial axes for both a polygonal object $X$ and its (infinite) complement $\bar{X}$, we will consider only a part of $\bar{X}$ bounded, but "sufficiently large" with respect to $X$, so that the notions and properties related to quasi-regularity remain unaltered. For instance one can intersect $\bar{X}$ with a square centered on $X$ with a size as large as required by the user, which will correspond in general to the finite support of a digital image.
} 

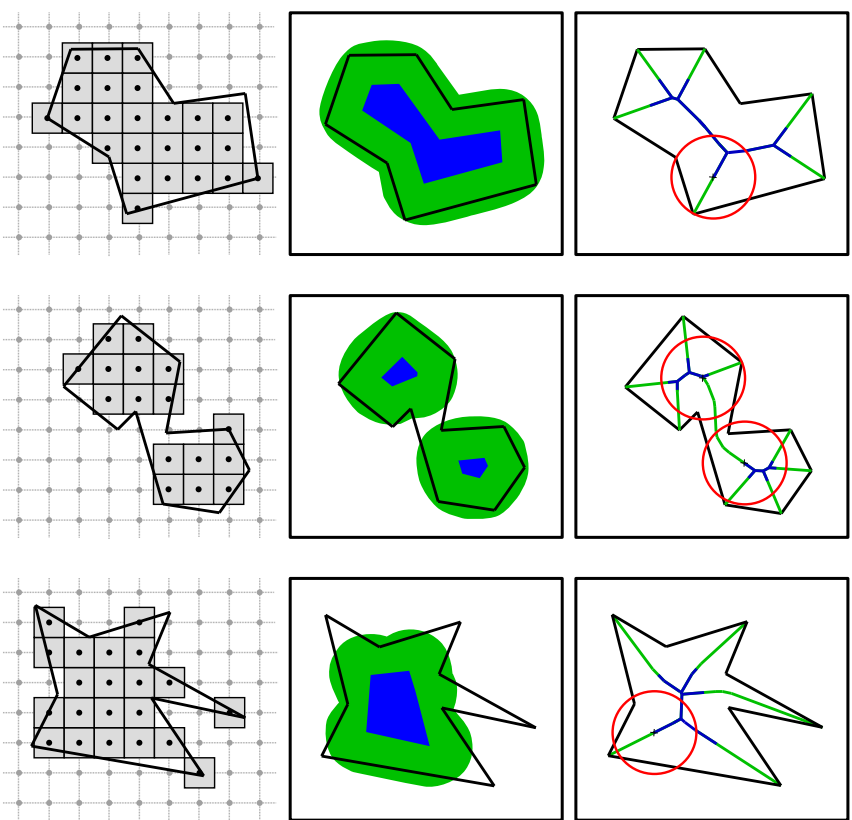

Fig. 2: Examples of quasi-regular and non-quasi-regular polygons. First column: the polygon $X_{i} \subset \mathbb{R}^{2}$ surrounded by a black polygonal line and its Gauss digitization $\mathrm{X}_{i}=X_{i} \cap \mathbb{Z}^{2}$ (grey pixels). $X_{1}$ and $\mathrm{X}_{1}$ have the same topology; this is not the case for $X_{2}$ and $\mathrm{X}_{2}$ (resp. $X_{3}$ and $\mathrm{X}_{3}$ ). Second column: quasi-regularity analysis based on Def. 11, in blue: $X_{i} \ominus B_{1}$ (condition $(i)$ ), in green: $X_{i} \ominus B_{1} \oplus B_{\sqrt{2}}$ (condition (iii)). Only $X_{1}$ is quasi-regular, it satisfies $(i)$ and $(i i i) ; X_{2}$ satisfies neither $(i)$ nor $(i i i) ; X_{3}$ satisfies (i) but not $($ iii $)$. Third column: quasi-regularity analysis based on Prop. 6, in blue: $\mathcal{M}_{1}(X)$, in green: $\mathcal{M}_{0}^{1}(X)$, in red: some balls $B(y, \sqrt{2})$ for certain points $y$ with $r(y)=1 . X_{1}$ satisfies Prop. 6 . $X_{2}$ does not $\left(\mathcal{M}_{1}\left(X_{2}\right)\right.$ is not connected); $X_{3}$ does not $((\mathcal{P})$ is not satisfied).

Proposition 6 provides sufficient conditions for establishing the quasi-regularity of a polygonal object $X$; see Fig. 2 They are not necessary conditions because $X$ may be quasi-regular even if $(\mathcal{P})$ is not satisfied for some of the connected components of $\mathcal{M}_{0}^{1}(X)$ or $\mathcal{M}_{0}^{1}(\bar{X})$. Such cases, however, correspond to tortuous shapes of $X$, which are rarely seen in usual applications. In other words, Prop. 6 provides us with a reasonable way of determining that a polygonal object is quasi-regular.

\section{QUASI-REGULARITY VERIFICATION METHOD}

Based on Prop. 6, we present hereafter a method to assess the quasiregularity of a polygonal object $X \subset \mathbb{R}^{2}$ using the medial axes $\mathcal{M}(Y)$ (with $Y \in\{X, \bar{X}\}$ ). From Prop. 6 it consists of verifying the following two conditions:

(a) $\mathcal{M}(Y) \frown \mathcal{M}_{1}(Y)$

(b) $(\mathcal{P})$ holds for each connected component of $\mathcal{M}_{0}^{1}(Y)$.

The process is summarized in Alg. 1

By construction, the medial axis of a polygon is represented by a planar graph structure [13], where vertices are incident to edges whose embeddings in $\mathbb{R}^{2}$ are either straight segments or arcs of parabolas. The radius function $r(\cdot)$ along the straight segments and

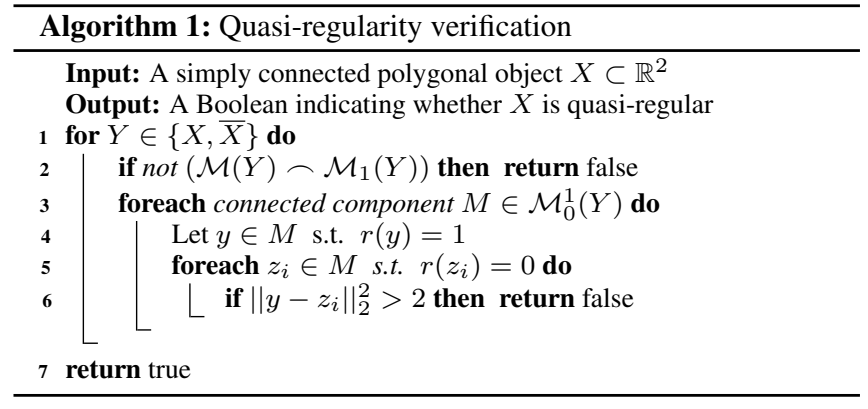

along the half-arcs of parabolas is monotonic. In this work, we use the package Segment Delaunay Graphs, proposed in CGAL [14] for exact computation of the medial axis of polygons. It allows us to extract the analytic expression for each graph edge as well as the associated radius function $r(\cdot)$. This is useful for computing $\mathcal{M}_{1}(Y)$ from $\mathcal{M}(Y)$ and finding the unique point $y$ of each connected component $M$ of $\mathcal{M}_{0}^{1}(Y)$. From the graph structure of $\mathcal{M}(Y)$, we can also extract the $k$ vertices $z_{i}, 1 \leq i \leq k$, for each $M$.

In order to verify $(a)$ the homotopy-type preservation between $\mathcal{M}(Y)$ and $\mathcal{M}_{1}(Y)$ (Line 2 of Alg. 11), we rely on the Euler characteristics $\chi=b_{0}-b_{1}$ where $b_{0}$ (resp. $b_{1}$ ) is the number of connected components (resp. cycles) of the graph. On the one hand, $\mathcal{M}(X)$ is simply connected, i.e. $\chi(\mathcal{M}(X))=1-0=1$. On the other hand, $\mathcal{M}(\bar{X})$ is connected with one cycle, i.e. $\chi(\mathcal{M}(\bar{X}))=1-1=0$. By construction, any part $Z$ of $\mathcal{M}(Y)$ satisfies $b_{0}(Z) \geq b_{0}(\mathcal{M}(Y))$ and $b_{1}(Z) \leq b_{1}(\mathcal{M}(Y))$, thus $\chi(Z) \geq \chi(\mathcal{M}(Y))$, and we have $\chi(Z)=\chi(\mathcal{M}(Y))$ iff $b_{0}$ and $b_{1}$ are the same for both, i.e. $Z$ and $\mathcal{M}(Y)$ have the same homotopy type. It is then sufficient to check $\chi$, which can be computed as $\chi=|V|-|E|$, where $V$ and $E$ are the sets of vertices and edges of (the graph structure of) the medial axis, respectively.

In order to verify $(b)$, i.e. that the property $(\mathcal{P})$ holds for every connected component $M \subseteq \mathcal{M}_{0}^{1}(Y)$, it is sufficient to evaluate the square of the Euclidean distance between the point $y \in M \cap \mathcal{M}_{1}^{1}(Y)$ and any of the points $z_{i} \in M \cap \mathcal{M}_{0}^{0}(Y)$, as stated in Line 6 of Alg. 1 .

Computing the medial axes has a complexity of $\mathcal{O}\left(n \log ^{2} n\right)$, with $n$ is the number of vertices of the polygon [15]. Checking $(a)$ has a complexity $\mathcal{O}(m)$, with $m$ the number of vertices of the medial axes, which is in the same order as $n$. Thus it can be also written as $\mathcal{O}(n)$. As checking $(b)$ has a complexity $\mathcal{O}(n)$, the overall complexity is then $\mathcal{O}\left(n \log ^{2} n\right)$.

\section{APPLICATION CASES}

Quasi-regularity characterized in Def. 1 provides a theoretical way of guaranteeing the preservation of homotopy type between a continuous object $X \subset \mathbb{R}^{2}$ and its digital analogue $\mathrm{X}=\mathcal{D}(X) \subset \mathbb{Z}^{2}$ obtained by Gauss digitization $\mathcal{D}$. From a practical point of view, Prop. 6 and the induced Alg. 1 provide an algorithmic way of assessing the quasi-regularity of $X \subset \mathbb{R}^{2}$ whenever $X$ is a (simply connected) polygonal object.

We can then consider quasi-regularity as a topological tool which is useful when a polygonal object has to be digitized. This is especially the case during rasterization procedures [16], which are frequent and crucial tasks, e.g. in computer graphics applications or digital image construction.

Beyond such polygon-to-pixel applications, quasi-regularity can be also useful for pixel-to-pixel applications, in particular when con- 
sidering digital geometric transformations. Indeed, defining "correct" geometric transformations from $\mathbb{Z}^{2}$ to $\mathbb{Z}^{2}$ is not a trivial task. Even for simple transformations, such as rigid motions (i.e. rotations composed with translations), this correctness is hard to reach, especially with regard to topology preservation [17, 18].

The notion of quasi-regularity can allow us to tackle this issue by handling such polygon-to-pixel procedures via pixel-to-polygonto-pixel strategies. Given a digital object $X \subset \mathbb{Z}^{2}$ and a (continuous) rigid motion $\mathfrak{R}: \mathbb{R}^{2} \rightarrow \mathbb{R}^{2}$, a common problem is to determine a digital object $\mathrm{X}_{\mathfrak{R}} \subset \mathbb{Z}^{2}$ that fits at best the object $\mathfrak{R}(\mathrm{X})=\{\mathfrak{R}(x) \mid$ $x \in \mathrm{X}\}$ which is a subset of $\mathbb{R}^{2}$ but most of the time not of $\mathbb{Z}^{2}$.

A possible solution relies on two steps: (1) defining a polygon $P(\mathrm{X})$ which is a relevant representation of $\mathrm{X}$ and (2) digitizing the image of this of polygon $P(\mathrm{X})$ after the rigid motion. More formally, we may compute:

$$
\mathrm{X}_{\mathfrak{R}}=\mathcal{D}(\mathfrak{R}(P(\mathrm{X})))
$$

Step (1) is a pixel-to-polygon process, whereas Step (2) is a polygonto-pixel one (actually a rasterization process).

The choice of $P(\mathrm{X})$ from $\mathrm{X}$ is of course, not unique. In our experiments, we considered that a "good" polygonalization should be reversible (i.e. $\mathcal{D}(P(\mathrm{X}))=\mathrm{X}$ ), preserve the topology (a simply connected digital object should lead to a simple polygon) and should lead to a polygon with rational-coordinate vertices.

Since we have $\mathrm{X} \frown P(\mathrm{X})$ by construction and $P(\mathrm{X}) \frown$ $\mathfrak{R}(P(\mathrm{X}))$ by definition, it is sufficient to guarantee that $\mathfrak{R}(P(\mathrm{X}))$ (or equivalently, $P(\mathrm{X})$ ) is quasi-regular to ensure that Eq. [15 defines a homotopy-type preserving digital transformation between the digital objects $\mathrm{X}$ and $\mathrm{X}_{\mathfrak{R}}$.

\section{EXPERIMENTS}

In our experiments, we mainly dealt with the digital rigid motion problem of Eq. 15]. Without loss of generality we considered rigid motions $\mathfrak{R}$ with rational values for their translation part and rotation angles built from Pythagorean triples [19]. This choice was not penalizing, due to the high density of such rigid motions. In addition, it allowed us to maximize the parts of the experiments carried out with exact calculation (and thus without numerical errors). Regarding the polygonalization, we considered the approach based on [5, 20]. For the digitization step, we used a ray-tracing based approach [21], namely the crossing number algorithm [22], to compute the Gauss digitization of the transformed polygon.

Some experimental results are illustrated in Fig. 3 Three complex digital objects $\mathrm{X}$ are represented by polygons $P(\mathrm{X})$ satisfying the required properties (reversibility, topology). These polygons are then proved quasi-regular thanks to Alg. 11. via their medial axis analysis. Their images by rigid motion can then be computed from Eq. 15. with guarantees of homotopy-type preservation.

Reversely, Fig. 4 illustrates the possible topological consequences of non-quasi-regularity. One can observe two polygonal objects that are not quasi-regular. The digital objects induced by their Gauss digitization present topological properties that vary depending on the rigid motion applied beforehand, emphasizing the non-necassary preservation of the homotopy type.

\section{CONCLUSION AND PERSPECTIVES}

In this article, we proposed an algorithmically tractable way of verifying the quasi-regularity of continuous objects consisting of simple polygons, based on the analysis of their medial axes. This allows, in particular, to guarantee topological preservation properties when
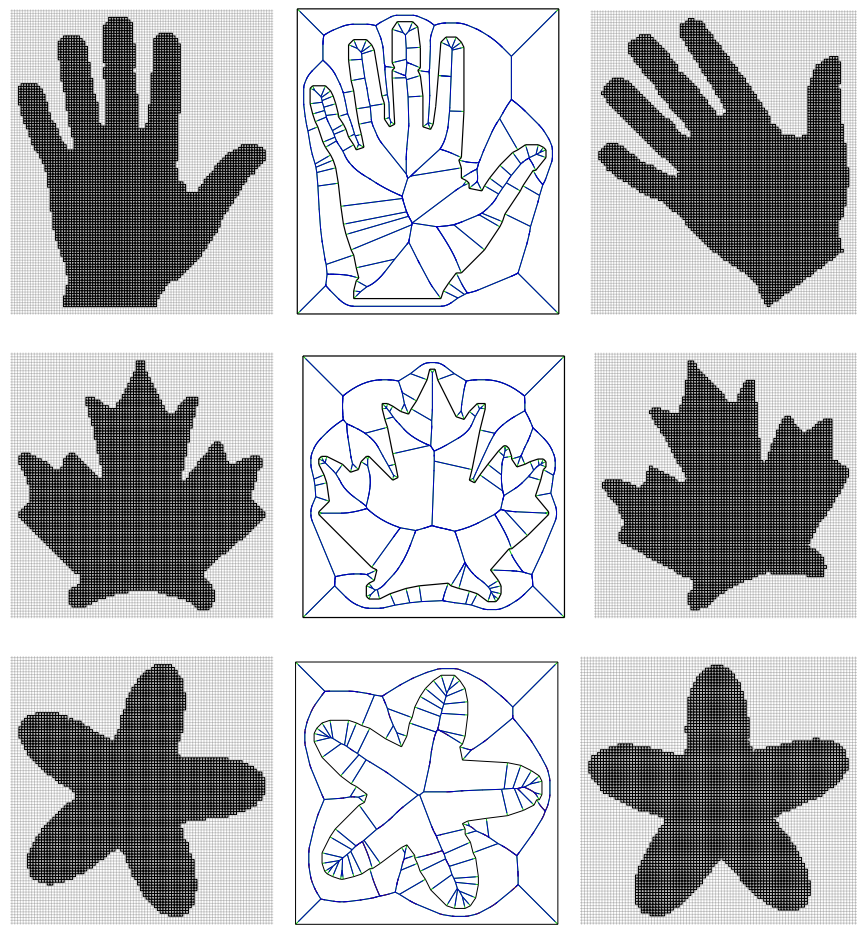

Fig. 3: Left: input digital objects $X \subset \mathbb{Z}^{2}$. Middle: polygonal representations $P(\mathrm{X})$ in black, and the induced medial axes, that allow to prove the quasi-regularity from Alg. 1. Right: resulting digital objects $X_{\mathfrak{R}}$ obtained from Eq. (15) for various rigid motions $\mathfrak{R}: \mathbb{R}^{2} \rightarrow \mathbb{R}^{2}$. The homotopy-type of $\mathrm{X}_{\mathfrak{R}}$ is then equal to that of $\mathrm{X}$.
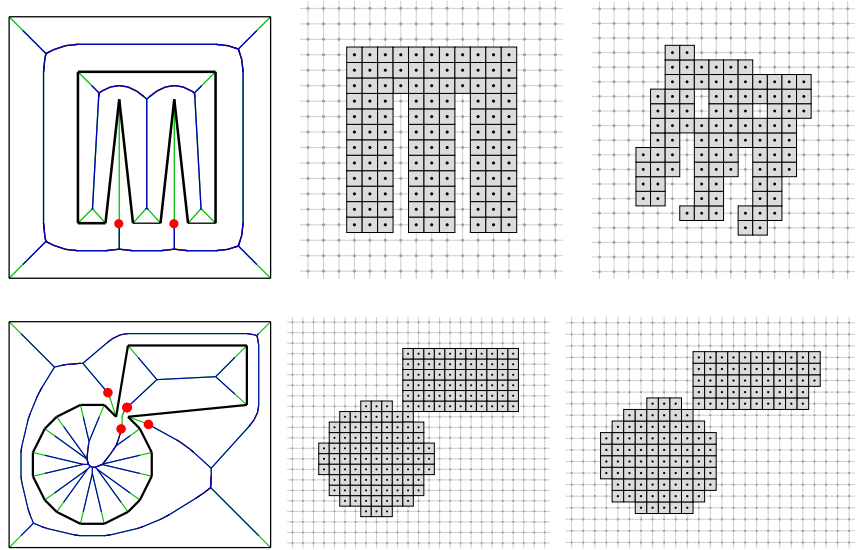

Fig. 4: Left: polygonal objects $P$ (in black) which do not satisfy Prop. 6 In blue, $\mathcal{M}_{1}(P)$. In green, $\mathcal{M}_{0}^{1}(P)$. In red, the points where $(\mathcal{P})$ is not satisfied or where disconnections occur. Middle: digital objects $\mathrm{X}$ obtained by Gauss digitization of $P$ with the same homotopy-type as $P$. Right: digital objects $\mathrm{X}$ obtained by Gauss digitization of $P$ (up to a topology-preserving rigid motion) with a different homotopy-type than $P$.

carrying out non-trivial operations such as rasterization or digital geometric transformations. Perspective works will consist of evaluating quasi-regularity for more complex objects, e.g. hierarchies of nested polygons. Longer term research will also deal with potential extensions of this approach to higher dimensions. 


\section{REFERENCES}

[1] R. Klette and A. Rosenfeld, Digital Geometry: Geometric Methods for Digital Picture Analysis, Elsevier, Amsterdam, Boston, 2004.

[2] T. Pavlidis, Algorithms for Graphics and Image Processing, Berlin: Springer, and Rockville: Computer Science Press, 1982.

[3] P. Stelldinger and K. Terzic, "Digitization of non-regular shapes in arbitrary dimensions," Image and Vision Computing, vol. 26, no. 10, pp. 1338-1346, 2008.

[4] P. Ngo, Y. Kenmochi, I. Debled-Rennesson, and N. Passat, "Convexity-preserving rigid motions of 2D digital objects," in Discrete Geometry for Computer Imagery (DGCI), Proceedings, 2017, pp. 69-81.

[5] P. Ngo, N. Passat, Y. Kenmochi, and I. Debled-Rennesson, "Geometric preservation of 2D digital objects under rigid motions," Journal of Mathematical Imaging and Vision, vol. 61, no. 2, pp. 204-223, 2019.

[6] P. Ngo, N. Passat, Y. Kenmochi, and I. Debled-Rennesson, "Convexity invariance of voxel objects under rigid motions," in International Conference on Pattern Recognition (ICPR), Proceedings, 2018, pp. 1157-1162.

[7] L. Mazo, N. Passat, M. Couprie, and C. Ronse, "Digital imaging: A unified topological framework," Journal of Mathematical Imaging and Vision, vol. 44, no. 1, pp. 19-37, 2012.

[8] A. Rosenfeld, "Digital topology," The American Mathematical Monthly, vol. 86, no. 8, pp. 621-630, 1979.

[9] J. Serra, Image Analysis and Mathematical Morphology, Academic Press, Inc., USA, 1983.

[10] L. J. Latecki, U. Eckhardt, and A. Rosenfeld, "Well-composed sets," Computer Vision and Image Understanding, vol. 61, no. 1, pp. 70-83, 1995.

[11] H. Blum, "A transformation for extracting new descriptors of shape," in Models for the Perception of Speech and Visual Form, Weiant Wathen-Dunn, Ed., pp. 362-380. MIT Press, Cambridge, 1967.

[12] A. Lieutier, "Any open bounded subset of $\mathrm{R}^{\mathrm{n}}$ has the same homotopy type as its medial axis," Computer-Aided Design, vol. 36, no. 11, pp. 1029-1046, 2004.

[13] F. P. Preparata, "The medial axis of a simple polygon," in Mathematical Foundations of Computer Science (MFCS), Proceedings, 1977, pp. 443-450.

[14] M. Karavelas, "2D segment Delaunay graphs," in CGAL User and Reference Manual. CGAL Editorial Board, 5.2 edition, 2020.

[15] M. I. Karavelas, "A robust and efficient implementation for the segment Voronoi diagram," in International Symposium on Voronoi Diagrams in Science and Engineering (ISVD), Proceedings, 2004, pp. 51-62.

[16] J. Pineda, "A parallel algorithm for polygon rasterization," in Annual Conference on Computer Graphics and Interactive Techniques, (SIGGRAPH), Proceedings, 1988, pp. 17-20.

[17] P. Ngo, Y. Kenmochi, N. Passat, and H. Talbot, "Topologypreserving conditions for 2D digital images under rigid transformations," Journal of Mathematical Imaging and Vision, vol. 49, no. 2, pp. 418-433, 2014.
[18] P. Ngo, N. Passat, Y. Kenmochi, and H. Talbot, "Topologypreserving rigid transformation of 2D digital images," IEEE Transactions on Image Processing, vol. 23, no. 2, pp. 885-897, 2014.

[19] W. S. Anglin, "Using Pythagorean triangles to approximate angles," American Mathematical Monthly, vol. 95, pp. 540541, 1988.

[20] T. P. Nguyen and I. Debled-Rennesson, "A discrete geometry approach for dominant point detection," Pattern Recognition, vol. 44, no. 1, pp. 32-44, 2011.

[21] M. Vandewettering, E. Haines, E. J. Kalenda, R. Parent, S. Uselton, Z. Andersson, and B. Holloway, "Point in polygon, one more time...," Ray Tracing News, vol. 3, no. 4, 1990.

[22] M. Shimrat, "Algorithm 112: Position of point relative to polygon," Communications of the ACM, vol. 5, no. 8, 1962. 